\title{
Acrolein Contributes to Human Colorectal Tumorigenesis Through Activation of RAS/MAPK Pathway
}

Hong-Chieh Tsai

Chang Gung Memorial Hospital Linkou Main Branch: Chang Gung Memorial Hospital Han-Hsing Tsou

National Yang-Ming University

Chun-Chi Lin

Taipei Veterans General Hospital

Shao-Chen Chen

National Yang-Ming University

Hsiao-Wei Cheng

National Yang-Ming University

Tsung-Yun Liu

National Yang-Ming University

Wei-Shone Chen

Taipei Veterans General Hospital

Jeng-Kai Jiang

Taipei Veterans General Hospital

Shung-Haur Yang

Taipei Veterans General Hospital

Shih-Ching Chang

Taipei Veterans General Hospital

Hao-Wei Teng

Taipei Veterans General Hospital

Hsiang-Tsui Wang ( $\nabla$ htwang01@ym.edu.tw )

Department of Pharmacology, National Yang-Ming University, Taipei, Taiwan https://orcid.org/00000003-4443-0670

\section{Research}

Keywords: Acrolein, colorectal cancer, high fat diet, RAS/MAPK pathway

Posted Date: January 8th, 2021 
DOI: https://doi.org/10.21203/rs.3.rs-139690/v1

License: (c) (1) This work is licensed under a Creative Commons Attribution 4.0 International License. Read Full License 


\section{Abstract}

Background. Colorectal cancer (CRC) is one of the most well-known malignancies with high prevalence and poor 5-year survival. Previous studies have demonstrated that intake of food rich in fat, and with low fiber content (as known as high-fat diet, HFD) is capable of increasing the odds of developing CRC. Acrolein, an IARC group 2A carcinogen, can be formed by thermal treatment of animal and vegetable fats, carbohydrates and amino acids through Maillard reaction. Also, acrolein has been shown to be produced from microbial glycerol metabolism in human gut. Consequently, humans are at risk of acrolein exposure through consumption of foods rich in fat. However, whether acrolein contributes to HFD-induced CRC tumorigenesis remains elusive.

Methods. The effect of acrolein in oncogenic transformation was analyzed using NIH/3T3 cells with xenograft tumorigenesis mice models. Furthermore, cDNA microarray analysis with Ingenuity Pathway Analysis (IPA) was performed in acrolein-transformed NIH/3T3 cells. Finally, acrolein-induced DNA damages (Acr-dG) were analyzed in tumor tissues and normal epithelial of CRC patients using immunohistochemical analysis. The levels of Acr-dG adducts were associated with tumor characteristics and CRC patients' survival using Chi-Square analysis and Kaplan Meier survival analysis, respectively.

Results. In this present study, we found that acrolein induced oncogenic transformation including faster cell cycling, proliferation, soft agar formation, sphere formation, cell migration in NIH/3T3 cells. Using xenograft tumorigenicity assays, the acrolein-transformed NIH3T3 clone formed tumors whereas no tumors were observed in mice inoculated with NIH3T3 parental cells. In addition, RAS/MAPK pathway contributing to colon tumorigenesis was activated in NIH/3T3 Acr-clone using CDNA microarray analysis with IPA. Finally, Acr-dG adducts were higher in CRC tumor tissues compared to normal epithelial cells in CRC patients. Intriguingly, CRC patients with higher Acr-dG adducts have better prognosis.

Conclusions. Taken together, this is the first study to demonstrate that acrolein is important in oncogenic transformation through activating RAS/MAPK signaling pathway contributing to colon tumorigenesis. Thus, acrolein might be a novel target for early detection, prevention and treatment of tumors in the future.

\section{Background}

Colorectal cancer (CRC) is the third most frequent neoplasm worldwide (www.wcrf.org). In spite of the fact that diagnosis and therapy have advanced significantly over the most recent ten years, its prevalence is rising, and the 5-year survival rate is as yet poor (1). CRC turns into a significant issue for healthcare in Asian countries with a 2-4 fold increase in the incidence during decades ago. It emerges from benign neoplasms and develops into adenocarcinomas through a stepwise histological progression sequence, continuing from either adenomas or hyperplastic polyps/serrated adenomas. Genetic modifications have been related with specific steps in this adenoma-carcinoma sequence and are believed to drive the histological progression of CRC $(2,3)$. It arises from an association of genetic and environmental factors, 
and it is identified with multiple cell signaling pathways, such as Wnt, epidermal growth factor receptor / mitogen-activated protein kinase (EGFR/ MAPK), tumor protein 53 (TP53), phosphoinositide3-kinase (PI3K), and transforming growth factor beta (TGFB)/SMAD $(4,5)$. Epidemiologic studies have shown that lifestyle and dietary habits impact the danger of developing CRC (6). In particular, the intake of foods rich in fat, and with low fiber content (as known as high-fat diet (HFD) or Western-style diet) is fit for increasing the odds of developing CRC (7-9).

Acrolein (2-propenal), the most reactive $\alpha, \beta$-unsaturated aldehydes, is a highly mutagenic and highly oxidizing environmental toxin (10). The most well studied source of acrolein exposure is through tobacco smoking, which has been shown to be associated with oral, lung and bladder cancer (11-16). IARC Working groups re-evaluated acrolein as probably carcinogenic to humans (Group 2A) on the basis of sufficient evidence of carcinogenicity in experimental animals and strong mechanistic evidence (17). Nonetheless, its dietary exposure and consequences is under investigated. Acrolein can be formed from carbohydrates, vegetable oils and animal fats, amino acids during preparation of foods (18). It is formed during the Maillard reaction as a result of the conversion of amino acids $(19,20)$ and the oxidative deamination of polyamines (21). Likewise acrolein could be detected in the emissions of varieties of heated or overheated cooking oils, and as such is found abundantly in fried food such as French fries (22). Also, recent reports demonstrated that gut microbial glycerol metabolism prompting the formation of reuterin, which is an additional source of endogenous acrolein (23). Reuterin is an antimicrobial multicomponent system comprising of 3-hydroxypropionaldehyde, its dimer and hydrate, and furthermore acrolein. Our recent studies have shown that exposure of acrolein from consuming fried food influences local oral cavity homeostasis (24). Consequently, humans are in danger of acrolein exposure through consumption of food rich in high fat $(25,26)$.

Although the association between HFD and CRC risk has been known for quite a while (27-29), the exact mechanisms underlying the HFD-induced colon cancer risk and recurrence have remained unclear. The mind boggling connections of dietary components with one another and with metabolism make it hard to specifically recognize the components in HFD which might cause CRC (8). Since acrolein can be produced during preparation of foods (18), we aim to investigate the role of acrolein in CRC tumorigenesis. In this present study, we determined the effect of acrolein in oncogenic transformation using NIH/3T3 cells with xenograft tumorigenesis mice models. Furthermore, cDNA microarray analysis with Ingenuity Pathway Analysis (IPA) was performed in acrolein-transformed NIH/3T3 cells. Finally, acrolein-induced DNA damages (Acr-dG) were analyzed in tumor tissues and normal epithelial of CRC patients and the levels of Acr-dG adducts were associated with tumor characteristics and CRC patients' survival.

\section{Methods}

Cell culture and acrolein treatment. Mouse fibroblast cell lien (NIH/3T3) and human normal colorectal cell CCD 841 CoN (ATCC 8 CRL-1790 ${ }^{\text {TM}}$ ) were purchased from ATCC and maintained in Dulbecco's Modified Eagle Medium (DMEM) supplemented with 10\% BCS and 15\% FBS, respectively. Acrolein stock solution 
(Sigma-Aldrich) was prepared freshly before use. Cells at 70\% confluency were treated with different concentrations of acrolein $(0-10 \mu \mathrm{M})$ in complete culture medium for $1-3$ months at $37^{\circ} \mathrm{C}$ in the dark and acrolein-containing medium was changed every two days.

Cell proliferation assay. Cell proliferation was determined using a modified 3-(4,5- dimethylthiazol-2yl)-2,5-diphenyl tetrazolium (MTT; Sigma, St. Louis, MO) assay (30). Briefly, cells (1000/ well) were seeded in 96-well plates overnight, and measured every day for 7 days. The resulting formazan dissolved with DMSO were measured at $570 \mathrm{~nm}$ and results were presented as the percentage of the control values. All of these experiments were performed in triplicate and were repeated independently at least three times.

Flow cytometry analysis of cell cycle phases. Cells were washed twice in ice-cold PBS and fixed in icecold $70 \%$ ethanol for $30 \mathrm{~min}$ or overnight at $4{ }^{\circ} \mathrm{C}$. Cells were then washed in PBS and digested with DNase-free RNase A (50 U/ ml) at $37^{\circ} \mathrm{C}$ for $30 \mathrm{~min}$. Before flow cytometry analysis, cells were resuspended in $500 \mu \mathrm{l}$ propidium iodide (PI, $10 \mu \mathrm{g} / \mathrm{ml}$; Sigma) for DNA staining. PI staining was used to measure for cell cycle status using a Becton-Dickinson FACScan instrument and Cell Quest software.

Soft agar colony formation assay. Soft agar colony formation assay will be performed as described previously (31). Briefly, a 3-ml aliquot of $1.2 \%$ agar in a culture medium will be plated in $60-\mathrm{mm}$ dishes. Then 1,000 cells of transformed malignant or untransformed cells will be mixed with $3 \mathrm{ml}$ of $0.35 \%$ agar in a medium and plated on the solidified bottom agar. When the top agar solidified, the dishes will be transferred to an incubator and cultured for 30 days. Two or three drops of the medium will be added to each dish three times a week. After culturing for 30 days, the visible cell colonies will be photographed and counted.

Tumor sphere culture assay. Acrolein-transformed NIH/3T3 clones were trypsinized, and re-suspended at 1000 cells/ Ultra-Low Attachment 96-well Plate (Corninig) in culture medium containing 2 mM Lglutamine, N2 supplement, B27 supplement, $20 \mathrm{ng} / \mathrm{mL}$ hrEGF (Sigma), $20 \mathrm{ng} / \mathrm{mL}$ hrbFGF (Sigma) for two weeks. Fresh growth factors were added to the cells twice a week. Cumulative total numbers of cells from the spheroid cultures were calculated.

Cell migration assay. The cell migration assay was carried out in vitro using modified Boyden chambers with a Transwell apparatus (polycarbonate filters with 8-mm pores, Corning). Parental NIH/3T3 or $\mathrm{NIH} / 3 \mathrm{~T} 3$ Acr-clones ( $5 \times 10^{4} /$ well, 6 -well plates) was suspended in $500 \mu \mathrm{l}$ of growth medium and added into the upper chamber. The lower chamber contained $750 \mu$ growth medium supplemented with $10 \%$ FCS. After $24 \mathrm{~h}$ incubation at $37^{\circ} \mathrm{C}$ in a $5 \% \mathrm{CO}_{2}$ incubator, cells on the upper filter surface were removed by wiping with a cotton swab. Filters were then fixed in methanol and stained with crystal violet. Cells that migrated to the lower filter surface were be counted in six random fields under a microscope at 200x magnification. These experiments were performed in triplicates and will be repeated at least three times.

Immunoblotting analysis. Cells were washed twice with ice-cold PBS and lysed on ice for 20 minutes in radioimmunoprecipitation assay (RIPA) lysis buffer $(20 \mathrm{mM}$ Tris $\mathrm{HCl}, 150 \mathrm{mM} \mathrm{NaCl}, 1 \%$ (v/v) NP-40, 1\% 
(w/v) sodium deoxycholate, $1 \mathrm{mM}$ Ethylenediaminetetraacetates (EDTA), $0.1 \%$ (w/v) sodium dodecyl sulfate polyacrylamide (SDS) plus protease and phosphatase inhibitors). Lysates were then centrifuged at 13,200 rpm for $10 \mathrm{~min}$, and the protein concentrations of supernatant were determined by $\mathrm{BCA}^{\mathrm{Tm}}$ Protein Assay Kit. Protein samples $(30 \mu \mathrm{g})$ were run on 8-100\% SDS-polyacrylamide gel electrophoresis and then transferred onto a polyvinylidene difluoride (Bio-Rad, U.S.A.) at $90 \mathrm{~V}$ for $120 \mathrm{~min}$. Proteins were transferred onto nitrocellulose membranes (Bio-Rad). Blots were probed with primary antibodies overnight at $4{ }^{\circ} \mathrm{C}$. Primary antibodies included: P-EGFR (Tyr1148, 1:1000, Cell signaling \#4404); EGFR (1:1000, Cell signaling\#2232); RAS (1:1000, Cell signaling \#3965); p-AKT (1:1000, Cell signaling\#4058); AKT (1:1000, Cell signaling\#4685); P-p44/42 MAPK (Erk1/2) (Thr202/Tyr204) (1:1000, Cell signaling\#9101); p44/42 MAPK (Erk1/2) (Thr202/Tyr204) antibody \#9102; Cyclin D1 (1:1000, Cell signaling\#2978); c-myc (1:500, Santa cruz, sc-42). After primary antibody incubation, the membrane were washed and incubated with a horseradish peroxidase-conjugated secondary IgG (1:3,000; Millipore) for $1 \mathrm{~h}$ at room temperature. Immunoreative bands were detected using Amersham Enhanced Chemiluminescence (Amersham Pharmacia Biotech, Piscataway, NJ, U.S.A.). The bound primary and secondary antibodies were stripped by incubating the membrane in stripping buffer (100 mM 2mercaptoethanol, $2 \%$ SDS) for $30 \mathrm{~min}$ at room temperature. The membrane was then re-probed with GAPDH (1:1000, Cell signaling, \#5174).

Xenograft mouse model. Fifteen 6-week-old male Balb/c nude mice, weighing 25-30 g, were used. All animal experiments were approved by the Institutional Animal Care and Use Committee of National YangMing University and carried out according to the Guidelines for Animal Research of National Yang-Ming University (IACUC\#1070208rr). Tumors were induced by injecting acolein-transformed NIH/3T3 cells ( $5 \times$ $10^{6}$ in $100 \mu \mathrm{lPBS}$ ) subcutaneously into the right axillary fossa of mice. To obtain the tumor growth curve, daily measurement of tumor was performed. Perpendicular diameter with a digital caliper and volumes were calculated by (length $\mathrm{x}$ width2)/2. Body weight was also assessed twice weekly. Tumor samples were collected after sacrifice. Each sample was cut in halves; one half was preserved in $4 \%$ paraformaldehyde and one half was flash frozen in liquid nitrogen and stored at $-80^{\circ} \mathrm{C}$ until further use.

RNA isolation and cDNA Microarray analysis. The total RNA was isolated from cells TRIzol® Reagent (Thermo Fisher Scientific), according to the manufacturer's instructions. RNA samples were quantified using an ND-1000 spectrophotometer (NanoDrop Technologies, Wilmington, U.S.A.) and the quality was using an Agilent 2100 Bioanalyzer with a Nanochip (Agilent, Santa Clara, CA) following the manufacture's instruction. The microarray hybridizations were performed using total RNA prepared from the $\mathrm{NIH} / 3 \mathrm{~T} 3$ and NIH/3T3 Acr-clone\#4. GeneChip Mouse Genome 4302.0 Affymetrix oligonucleotide Gene Chips (Affymetrix) were analyzed at the Microarray \& Gene Expression Analysis Core Facility (VYM Genome Research Center, National Yang-Ming University) according to the Affymetrix protocols. Microarray datasets were analyzed using Ingenuity Pathway Analysis (IPA version 57662101) (QIAGEN).

Collection of formalin-fixed paraffin-embedded (FFPE) tissues and tissue microarray collection of CRC patients. 18 CRC FFPE tissues with corresponded tissue microarray collected at Taipei Veterans General Hospital were recruited for participate in the study. For each case, tumor tissue and adjacent non-tumor 
tissue samples were surgically dissected and sent to the Department of Pathology of examination. Patients with a diagnosis of CRC were included in the present study. Our study protocol was approved by the Institutional Review Board of Taipei Veterans General Hospital (IRB\#2020-01-010BC).

Immunohistochemistry (IHC) analysis. for Acr-dG adduct and c-myc. For the tissue microarray (TMA), hematoxylin and eosin-stained sections from each paraffin-embedded, formalin-fixed block were used to define diagnostic areas, and a representative $0.6 \mathrm{~mm}$ core was obtained from each case and inserted in a grid pattern into a recipient paraffin block. Sections $(4 \mu \mathrm{m})$ were then deparaffinized in xylene and rehydrated in a descending ethanol series. To enhance immunoreactivity, sections were incubated in TRIS-EDTA, pH 6.0, and boiled for $12 \mathrm{~min}$. Endogenous peroxidase activity was eliminated by incubation in hydrogen peroxide. Incubation with primary antibodies for Acr-dG antibody (generated in house), c-myc (Santa Cruz, sc-40) was performed overnight at $4{ }^{\circ} \mathrm{C}$ in $1 \%$ BSA in phosphate buffer saline (PBS). Bound antibodies were visualized using the Envision Detection System (K500711; Dako Denmark A/S), and DAB (diaminobenzidine) was used as a chromogen. Omission of the primary antibody was served as a negative control. Positive controls (normal liver) were stained in parallel with each set of TMA studied. Assessment of Acr-dG and c-myc immunoexpression was performed by light microscopy at x400 magnification by a pathologist.

Statistical analyses. Descriptive statistics will be presented as the mean \pm standard deviation or as the number (percentage). Student's t-tests were used to determine statistical significance, and two-tailed Pvalues are shown. A minimum of three independent replicate experiments was performed to justify the use of statistical tests. Survival was analyzed using Kaplan Meier survival analysis, and the log rank test was used for comparison between the two groups. Multivariate analysis was performed using Chi-Square analysis. Statistical significance will be defined as a $p<0.05$. All analyses will be performed with the IBM SPSS Statistics software package, version 23.0.

\section{Results}

Acrolein treatment induced cell proliferation, anchorage-independent activity, spheroid formation ability and cell migration capacity.

To determine the potential of acrolein in oncogenic transformation, we treated NIH/3T3 with low dose of acrolein $\left(7.5 \mu \mathrm{M}, \mathrm{IC}_{10}\right)$ for one month and selected as NIH/3T3 Acr-clones, \#1-\#7 (Supplementary

Fig. 1A). Soft agar colony formation activity of these 7 clones were analyzed and the result showed that $\mathrm{NIH} / 3 \mathrm{~T} 3$ Acr-clone \#3 and \#4 formed more colony numbers than others (Supplementary Fig. 1B). Cell proliferation analysis showed that $\mathrm{NIH} / 3 \mathrm{~T} 3 \mathrm{Acr}-\mathrm{clone} \# 4$ (doubling time $=31.0 \mathrm{~h}$ ) has faster proliferation compared to parental cells (doubling time $=39.4 \mathrm{~h}$ ); however, $\mathrm{NIH} / 3 \mathrm{~T} 3 \mathrm{Acr}-\mathrm{clone} \# 3$ (doubling time = $55.0 \mathrm{~h}$ ) showed the opposite phenomenon (Fig. 1A, Supplementary Fig. 1C). Therefore, we selected $\mathrm{NIH} / 3 \mathrm{~T} 3 \mathrm{Acr}-\mathrm{clone} \# 4$ for the following analysis. Consistently, cell cycle analysis showed the ratio of $\mathrm{NIH} / 3 \mathrm{~T} 3$ Acr-clone\#4 in S phase was markedly higher than in parental cells (Fig. 1B), indicating that acrolein promotes S-phase DNA synthesis and accelerates cell proliferation. Anchorage independent 
activity (Fig. 1C) in NIH3T3 Acr-clone\#4 was also increased compared to parental NIH/3T3 cells using soft agar colony formation assay. Spheroid formation ability on ultra-low attachment plates of NIH3T3 Acr-clone\#4 was also enhanced (Fig. 1D). In addition, NIH3T3 Acr-clone\#4 showed enhanced migration capacity compared with NIH3T3-mock cells (Fig. 1E) using transwell assay. However, drug sensitivity of $\mathrm{NIH} 3 \mathrm{~T} 3$ Acr-clone\#4 toward chemotherapeutic agents such as oxaliplatin and 5-FU was similar to parental NIH/3T3 cells (Supplementary Fig. 2). These results suggest that acrolein increases cell cycle rate, proliferation, colony formation activity, spheroid formation ability and cell migration capacity.

\section{NIH3T3 Acr-clone\#4 formed tumors in xenografts nude mice.}

Our in vitro results indicated that acrolein can transform normal mouse NIH/3T3 fibroblasts into malignant cells. To confirm its tumorigenic potential, we performed in vivo studies of tumor xenografts in nude mice, using parental NIH3T3 as the negative control. NIH3T3 Acr-clone\#4 and parental NIH3T3 cells were injected subcutaneously into the right axillary fossa $\left(5 \times 10^{6}\right.$ cells/animal). Three weeks after injecting NIH/3T3 Acr-clone\#4 into nude mice, nodular neoplasms could be observed while tumors were obvious at 10 days, whereas the parental NIH/3T3 cells failed to form any tumors (Fig. 2A). Tumors formed by NIH/3T3 Acr-clone\#4 were observed and their volumes and growth curves calculated for 4 weeks after the tumors could be observed (Fig. 2B-C). These data further indicate that acrolein leads to oncogenic transformation in vivo.

\section{Acrolein induced RAS/MAPK signaling pathway in CRC tumorigenesis using Ingenuity Pathway Analysis (IPA).}

To determine the underlying mechanisms by which acrolein induced oncogenic transformation, cDNA microarray analysis with IPA was performed in NIH/3T3 Acr-clone\#4 (Fig. 3A). The results showed that four genes (Rnd1, Rras2, myc and PI3Kcb) involved in RAS/MAPK signaling pathway were upregulated in acrolein-transformed clone\#4 (NIH/3T3 Acr-clone\#4) (Fig. 3B). These results were confirmed using Western blot analysis (Fig. 3C). Furthermore, we also found that acrolein activated RAS/MAPK signaling pathway and increased c-myc in NIH/3T3 cell and human normal colon epithelium, CCD-841CoN in the time-dependent and dose dependent manner (Fig. 3D, 3E).

\section{Immunohistochemistry analysis of Acrolein-DNA (Acr-dG) levels in human colon cancers.}

Acrolein can react with DNA inducing modifications, which, if not repaired, can result in mutations and lead to cancer development. Acrolein has been shown to produce propano-2'-deoxyguanosine (Acr-dG) adducts in human cells $(15,32,33)$. It has been found that Acr-dG adducts are mutagenic and that they induce mainly $G$ to $T$ and $G$ to $A$ mutations $(15,33-42)$. To further investigate whether acrolein contributes to colon cancer formation, we analyzed Acr-dG adduct expression in CRC tissues and the normal epithelial cells adjacent to the tumor tissues using immunohistochemical (IHC) staining. The result showed that Acr-dG adduct levels were mainly located in nucleus and were higher in CRC tumor tissue compared to normal epithelial cells in 18 CRC patients (Fig. 4). Based on our CDNA microarray data, c-myc was upregulated in acrolein-transformed cell clones (Fig. 4). We further analyzed c-myc level 
CRC tissues and the normal epithelial cells in same patients using IHC staining (Supplementary Fig. 3). Similar to Acr-dG adduct levels, higher c-myc levels were observed in CRC tumor tissues compared to normal epithelial cells.

\section{Higher Acr-dG expression is associated with improved survival in CRC patients.}

We further evaluated the effect of Acr-dG expression on CRC characteristics and patient survival. The demographic data were shown in Table 1. Of 236 CRC patients, the majority of tumor type was adenocarcinoma $(211 / 236,89.4 \%)$ and advanced stage III and IV cancer was diagnosed in $64 \%$ $(151 / 236)$ of patients. The expression of Acr-dG was defined as low $(H$ score $<50)$ and high expression $(H$ score $\geq 50$ ) based on the results showing that Acr-dG levels in CRC tumor tissues and adjacent normal epithelia (Fig. 4C). Kaplan-Meier survival analysis revealed the median survival were 103.4 months for CRC patients with high Acr-dG levels and 74.3 months for CRC patients with low Acr-dG levels indicating high expression of Acr-dG in tumor tissue was associated with better CRC patient overall survival $(p=$ 0.003 , Fig. 5). In addition, high expression of Acr-dG was also inversely correlated with clinical stages and grades using Chi-square analysis (Table 1). Our data suggests that CRC patients with higher Acr-dG expression in tumor tissues is associated with better prognosis. 
Table 1

Clinical characteristics of GBM patients and Chi-Square analysis.

\begin{tabular}{|c|c|c|c|c|c|c|}
\hline & & \multicolumn{4}{|c|}{ Acr-dG levels } & \multirow{3}{*}{$\begin{array}{l}\mathrm{P} \\
\text { value }\end{array}$} \\
\hline & & \multicolumn{2}{|c|}{$\begin{array}{l}\text { Low } \\
\text { (h score }<50 \text { ) }\end{array}$} & \multicolumn{2}{|c|}{$\begin{array}{l}\text { High } \\
\text { (h score > = 50) }\end{array}$} & \\
\hline & & Count & $\%$ & Count & $\%$ & \\
\hline \multirow[t]{2}{*}{ Age } & $<70$ years & 56 & $53.8 \%$ & 83 & $62.9 \%$ & \multirow[t]{2}{*}{0.161} \\
\hline & $>=70$ years & 48 & $46.2 \%$ & 49 & $37.1 \%$ & \\
\hline \multirow[t]{2}{*}{ Sex } & Female & 40 & $38.5 \%$ & 44 & $33.3 \%$ & \multirow[t]{2}{*}{0.414} \\
\hline & Male & 64 & $61.5 \%$ & 88 & $66.7 \%$ & \\
\hline \multirow[t]{2}{*}{ Location } & Left & 43 & $41.3 \%$ & 60 & $45.5 \%$ & \multirow[t]{2}{*}{0.528} \\
\hline & Right & 61 & $58.7 \%$ & 72 & $54.5 \%$ & \\
\hline \multirow[t]{3}{*}{ Pathology } & Adenocarcinoma & 91 & $87.5 \%$ & 120 & $90.9 \%$ & \multirow[t]{3}{*}{0.430} \\
\hline & Carcinoma & 1 & $1.0 \%$ & 0 & $.0 \%$ & \\
\hline & $\begin{array}{l}\text { Mucinous } \\
\text { adenocarcinoma }\end{array}$ & 12 & $11.5 \%$ & 12 & $9.1 \%$ & \\
\hline \multirow{4}{*}{$\begin{array}{l}\text { Clinical Stage (AJCC } \\
\text { 6th) }\end{array}$} & I & 6 & $5.8 \%$ & 12 & $9.1 \%$ & \multirow[t]{4}{*}{$0.041 *$} \\
\hline & II & 26 & $25.0 \%$ & 41 & $31.1 \%$ & \\
\hline & III & 26 & $25.0 \%$ & 44 & $33.3 \%$ & \\
\hline & IV & 46 & $44.2 \%$ & 35 & $26.5 \%$ & \\
\hline \multirow[t]{2}{*}{ Grade } & Low & 90 & $86.5 \%$ & 126 & $95.5 \%$ & \multirow[t]{2}{*}{$0.015^{\star}$} \\
\hline & High & 14 & $13.5 \%$ & 6 & $4.5 \%$ & \\
\hline \multirow[t]{2}{*}{ Mucinous component } & No & 64 & $61.5 \%$ & 83 & $62.9 \%$ & \multirow[t]{2}{*}{0.833} \\
\hline & Yes & 40 & $38.5 \%$ & 49 & $37.1 \%$ & \\
\hline \multirow[t]{2}{*}{ LVSI $^{b}$} & No & 74 & $71.2 \%$ & 107 & $81.1 \%$ & \multirow[t]{2}{*}{0.074} \\
\hline & Yes & 30 & $28.8 \%$ & 25 & $18.9 \%$ & \\
\hline \multirow[t]{2}{*}{$\mathrm{PNI}^{\mathrm{C}}$} & No & 37 & $88.1 \%$ & 38 & $97.4 \%$ & \multirow[t]{2}{*}{0.109} \\
\hline & Yes & 5 & $11.9 \%$ & 1 & $2.6 \%$ & \\
\hline
\end{tabular}




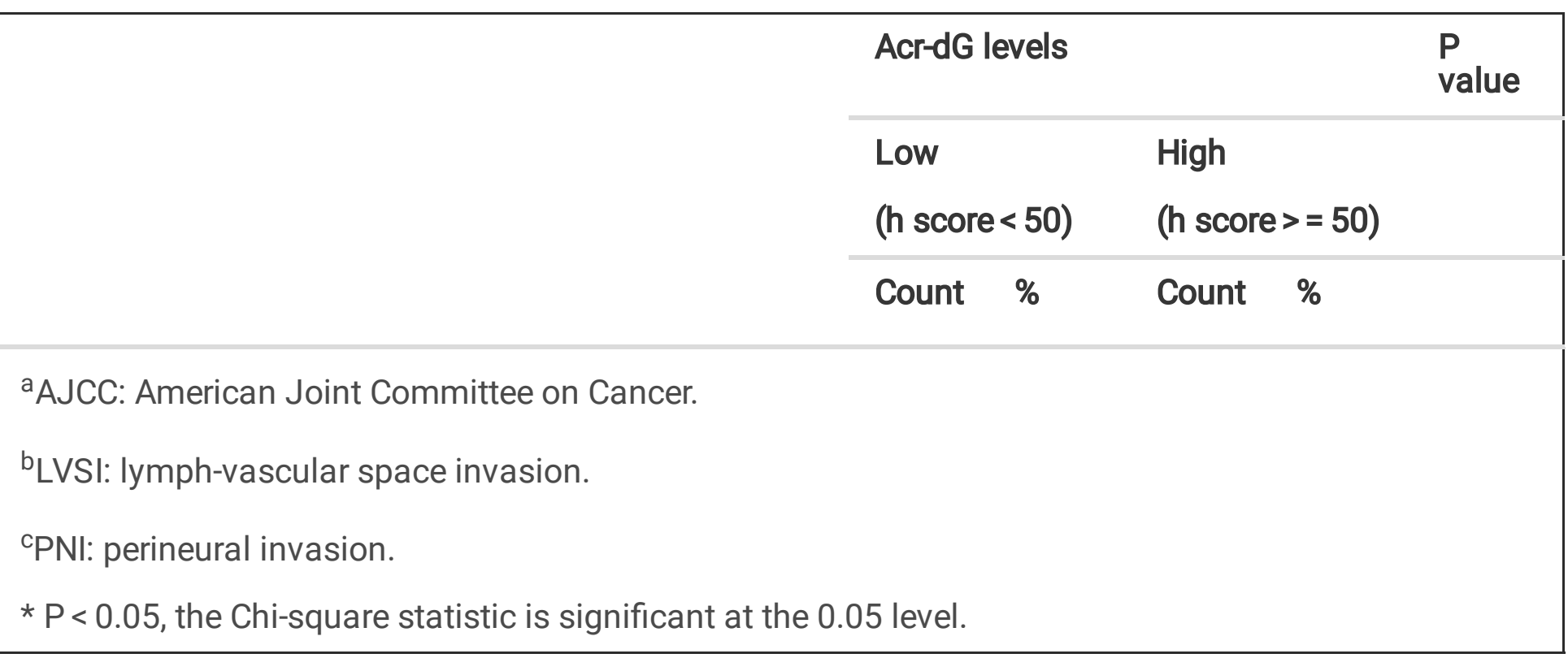

\section{Discussion}

Acrolein is the most reactive $a, \beta$-unsaturated aldehyde present in tobacco smoke, in ambient air pollution, and in some cooking oils heated to a high temperature (18). Acrolein was previously evaluated as group 3 carcinogen by the IARC Working Group in 1995; however, it was re-evaluated as probably carcinogenic to humans (Group 2A) (17). Our previous studies have also supported that acrolein is associated with oral, lung and bladder cancer $(12,13,15,16)$. Furthermore, our current studies have shown that individuals could expose to acrolein from consuming fried food (24). In spite of the fact that the association between HFD and CRC risk has been known for quite a while (27-29), the exact mechanisms underlying the HFDinduced colon cancer risk and recurrence remain unclear. In the present study, our results showed that acrolein induced oncogenic transformation in NIH/3T3 cells in vitro and in vivo. The underlying mechanism was through activation of RAS/MAPK pathway which contributes to colon carcinogenesis. Additionally, Acr-dG adducts were higher in CRC tumor tissues compared to normal epithelial cells in CRC patients. These results suggested that acrolein may contribute to colon tumorigenesis. Beside, slot blot analysis showed that increased Acr-dG levels in mice colon tissues with HFD for 24 weeks compared to mice with normal diet (Supplementary Fig. 4). Furthermore, we found that acrolein-protein conjugates (Acr-PC) were increased in colon tissues of mice fed with HFD for 4-24 weeks (Supplementary Fig. 5). These results indicated that HFD induced acrolein production in mice colon and acrolein may contribute to HFD-induced colon tumorigenesis.

In vivo exposure of acrolein in most situations is quite low and the effects may differ from those seen at acutely toxic doses $(10,43)$. Acrolein, the most reactive of the $a, \beta$-unsaturated aldehyde, rapidly binds to and deplete cellular nucleophiles such as glutathione, and also react with cysteine, histidine, and lysine residues of proteins and with nucleophilic sites in DNA (43-45). This reactivity is the basis for the cytotoxicity evident in all cells exposed to high concentrations of acrolein (46-49). On the other hand, acrolein inhibits cell proliferation without causing cell death at low doses (50-52). In this present study, we used sublethal dose of acrolein $(7.5 \mu \mathrm{M}, \mathrm{IC} 10)$ to expose $\mathrm{NIH} / 3 \mathrm{~T} 3$ cells for one months in order to 
mimic in vivo condition (Supplementary Fig. 1). The results showed acrolein was able to transform $\mathrm{NIH} / 3 \mathrm{~T} 3$ cells and NIH/3T3 Acr-clone \#3 and \#4 formed more colony numbers than others (Supplementary Fig. 1B). However, cell proliferation analysis showed the opposite phenomenon (Fig. 1A, Supplementary Fig. 1C). Furthermore, NIH/3T3 Acr-clone\#4 was able to form tumors whereas no tumors were observed in mice inoculated with $\mathrm{NIH} / 3 \mathrm{~T} 3$ parental cells as well as $\mathrm{NIH} / 3 \mathrm{~T} 3$ Acr-clone\#3 (data not shown) using xenograft mouse model (Fig. 2). The mechanisms underlying acrolein-induced cell transformation may be related to the ability of acrolein to deplete cellular thiols or other nucleophiles, and/or to effects on gene activation, either directly or subsequent to effects on redox-regulated transcription factors $(10,43)$. In order to explore the possible signaling involving in acrolein-induced oncogenic transformation, we used cDNA microarray with IPA analysis and results showed that RAS/MPAK pathway was the top of Canonical pathway analysis (Fig. 3A).

Previous studies have shown that alterations in EGFR-related Ras-Raf-MAPK and PI3K-Akt pathways are involved in the pathogenesis of up to $55 \%$ and $15 \%$ of CRC, respectively (53). Upregulation of c-myc protein plays an essential role in tumorigenesis through frequently altered kinase MAPK and RAS pathways in CRC (54). In this study, we found that acrolein upregulated RAS/MAPK pathway followed by overexpression of c-myc in both $\mathrm{NIH} / 3 \mathrm{~T} 3$ and colon cells, CCD-841 coN (Fig. 3). Consistently, increased cmyc expression was also observed in these CRC tumor tissues (Supplementary Fig. 3) along with higher Acr-dG adducts in these tumor tissues (Fig. 4). Acrolein is a highly reactive aldehyde reacting with $\mathrm{dG}$ of DNA to form Acr-dG adducts which were shown to be mutagenic $(15,33-42)$. It is unclear whether acrolein induced mutations in RAS/MAPK pathways. These results showed that acrolein may be involved in colon tumorigenesis and the underlying mechanism is possible through activation of RAS/MAPK pathway and upregulation of c-myc.

Interestingly, we found that CRC patients with higher Acr-dG expression in tumor tissues is associated with better prognosis (Fig. 5, Table 1). The possible explanation is that Acr-dG adducts is involved in the initiation of colon tumorigenesis; however, accumulating high amounts of Acr-dG adducts trigger cellular apoptosis. Acrolein can be produced through lipid peroxidation in fast dividing cells such as cancer cells (55). Our previous studies have shown that hypoxia induced acrolein production resulting in cellular apoptosis (56). In addition, acrolein induced cytotoxicity in colon cancer cell lines, SW480 and HCT116 (Supplementary Fig. 6). This may explain CRC patients with higher Acr-dG adduct levels were associated with better survival. However, the detailed mechanisms still need further investigation.

Major restriction of this study is that $\mathrm{NIH} / 3 \mathrm{~T} 3$ is a mouse fibroblast cell model and the genetic background may not be correlated with epithelia, though NIH/3T3 has been recognized as a cell line for tumorigenesis study in vitro and in vivo (17). We could not observe EGFR expression in NIH/3T3 which is similar to previous studies showing that $\mathrm{NIH} / 3$ T3 is lack of $\operatorname{EGFR}(57,58)$. Therefore, we tried to use a normal colon cell, CCD-841 CoN as a model and found that acrolein indeed induce activation of RAS/MAPK pathway which was similar to NIH/3T3 (Fig. 3E). In addition, we found acrolein increased phosphorylation of EGFR indicating activation of EGFR results in downstream RAS/MAPK pathway in 
CCD-841CoN. However, we were unable to select CCD-841CoN Acr-clones successfully due to low passage numbers of CCD-841CoN.

\section{Conclusions}

Taken together, we found that acrolein induced oncogenic transformation using NIH/3T3 cells with a xenograft mouse model through upregulation of RAS/MAPK pathway. Besides, higher acrolein-induced DNA damages (Acr-dG adducts) were observed in tumor tissues compared to adjacent normal epithelial cells in CRC patients. Interestingly, increased Acr-dG levels were associated with better prognosis of CRC patients. To our knowledge, this is the first study to show that acrolein is important in oncogenic transformation through activating RAS/MAPK signaling pathway contributing to colon carcinogenesis. Thus, acrolein might be a novel target for early detection, prevention and treatment of tumors in the future.

\section{Abbreviations}

Acr-dG

acrolein-induced DNA damages; Acr-clone\#4:acrolein-transformed clone\#4; CRC:colorectal cancer; EGFR:epidermal growth factor receptor; FFPE:formalin-fixed paraffin-embedded; HFD:high-fat diet; IHC:immunohistochemistry; IPA:Ingenuity Pathway Analysis; MAPK:mitogen-activated protein kinase; PI3K:phosphoinositide3-kinase:TP53:tumor protein 53; TMA:tissue microarray;

\section{Declarations}

Ethics approval. Our human study protocol was approved by the Institutional Review Board of Taipei Veterans General Hospital (IRB\#2020-01-010BC). All animal experiments were approved by the Institutional Animal Care and Use Committee of National Yang-Ming University and carried out according to the Guidelines for Animal Research of National Yang-Ming University (IACUC\#1070208rr).

Consent to participate. Not applicable.

Consent for publication. The authors gave consent for publication.

Availability of data and material. Not applicable.

Competing interests. The authors have no actual or potential competing interests.

Funding. This work was funded by grants from Ministry of Science and Technology, Taiwan [109-2320-B010-018 (H-T Wang)], Taiwan Clinical Oncology Research Foundation, Ministry of Health and Welfare, Taiwan (109-2314-B-075-081-MY3), Taipei Veterans General Hospital (110DHA0100397) and ChangGung Memorial Hospital [CMRPG3K1441 (H-C Tsai)]. The authors would like to acknowledge the support by the Biobank of Taipei Veterans General Hospital and the technical services provided by Microarray \& 
Gene Expression Analysis Core Facility of the National Yang-Ming University VGH Genome Research Center (VYMGC). The Gene Expression Analysis Core Facility is supported by National Research Program for Genomic Medicine (NRPGM), National Science Council.

Authors' contributions H-C. T., H-H T., S-C C., H-W. C., H-T W. performed experiments; C-C. L., W-S. C., J-K. J., S-H. Y., S-C. C., H-W. T. collected and analyzed clinical samples. T-Y. L, H-W T., H-T W. designed experiments and participated in manuscript writing.

\section{References}

1. Howlader N NA, Krapcho M et al. . SEER Cancer Statistics Review, 1975-2013. . Bethesda, MD, USA2016; Available from: http://seer.cancer.gov/csr/1975_2013/.GoogleScholar.

2. Waliszewski P. Controversies about the genetic model of colorectal tumorigenesis. Pol $\mathrm{J}$ Pathol. 1995;46(4):239-43. Epub 1995/01/01.

3. Fearon ER, Vogelstein B. A genetic model for colorectal tumorigenesis. Cell. 1990;61(5):759-67. Epub 1990/06/01.

4. Tiwari A, Saraf S, Verma A, Panda PK, Jain SK. Novel targeting approaches and signaling pathways of colorectal cancer: An insight. World J Gastroenterol. 2018;24(39):4428-35. Epub 2018/10/26.

5. Farooqi AA, de la Roche M, Djamgoz MBA, Siddik ZH. Overview of the oncogenic signaling pathways in colorectal cancer: Mechanistic insights. Semin Cancer Biol. 2019;58:65-79. Epub 2019/01/12.

6. Walther A, Johnstone E, Swanton C, Midgley R, Tomlinson I, Kerr D. Genetic prognostic and predictive markers in colorectal cancer. Nature reviews Cancer. 2009;9(7):489-99. Epub 2009/06/19.

7. Vargas AJ, Thompson PA. Diet and nutrient factors in colorectal cancer risk. Nutrition in clinical practice : official publication of the American Society for Parenteral and Enteral Nutrition. 2012;27(5):613-23. Epub 2012/08/16.

8. Nystrom M, Mutanen M. Diet and epigenetics in colon cancer. World J Gastroenterol. 2009;15(3):25763. Epub 2009/01/14.

9. Newmark HL, Yang K, Lipkin M, Kopelovich L, Liu Y, Fan K, et al. A Western-style diet induces benign and malignant neoplasms in the colon of normal C57Bl/6 mice. Carcinogenesis. 2001;22(11):18715. Epub 2001/11/08.

10. Moghe A, Ghare S, Lamoreau B, Mohammad M, Barve S, McClain C, et al. Molecular mechanisms of acrolein toxicity: relevance to human disease. Toxicol Sci. 2015;143(2):242-55.

11. Bein K, Leikauf GD. Acrolein - a pulmonary hazard. Molecular nutrition \& food research. 2011;55(9):1342-60. Epub 2011/10/14.

12. Tsou HH, Hu CH, Liu JH, Liu CJ, Lee CH, Liu TY, et al. Acrolein Is Involved in the Synergistic Potential of Cigarette Smoking- and Betel Quid Chewing-Related Human Oral Cancer. Cancer epidemiology, biomarkers \& prevention : a publication of the American Association for Cancer Research, cosponsored by the American Society of Preventive Oncology. 2019;28(5):954-62. Epub 2019/03/08. 
13. Lee HW, Wang HT, Weng MW, Chin C, Huang W, Lepor H, et al. Cigarette side-stream smoke lung and bladder carcinogenesis: inducing mutagenic acrolein-DNA adducts, inhibiting DNA repair and enhancing anchorage-independent-growth cell transformation. Oncotarget. 2015;6(32):33226-36. Epub 2015/10/03.

14. Zhang C, Zhang M, Pang X, Zhao Y, Wang L, Zhao L. Structural resilience of the gut microbiota in adult mice under high-fat dietary perturbations. The ISME journal. 2012;6(10):1848-57. Epub 2012/04/13.

15. Tang MS, Wang HT, Hu Y, Chen WS, Akao M, Feng Z, et al. Acrolein induced DNA damage, mutagenicity and effect on DNA repair. Molecular nutrition \& food research. 2011;55(9):1291-300. Epub 2011/06/30.

16. Feng Z, Hu W, Hu Y, Tang MS. Acrolein is a major cigarette-related lung cancer agent: Preferential binding at p53 mutational hotspots and inhibition of DNA repair. Proc Natl Acad Sci U S A. 2006;103(42):15404-9.

17. Greig RG, Koestler TP, Trainer DL, Corwin SP, Miles L, Kline T, et al. Tumorigenic and metastatic properties of "normal" and ras-transfected NIH/3T3 cells. Proc Natl Acad Sci U S A. 1985;82(11):3698-701. Epub 1985/06/01.

18. Richard H. Stadler DRL. Process-Induced Food Toxicants: Occurrence, Formation, Mitigation, and Health Risks. New Jersey Wiley; 2008.

19. Ferretti A FV. Lactose casein (Maillard) browning system: volatile components. J Agric Food. 1971;19:5.

20. Alarcon RA. Formation of acrolein from various amino-acids and polyamines under degradation at 100 degrees C. Environ Res. 1976;12(3):317-26.

21. Lee Y, Sayre LM. Reaffirmation that metabolism of polyamines by bovine plasma amine oxidase occurs strictly at the primary amino termini. J Biol Chem. 1998;273(31):19490-4.

22. Osorio VM, de Lourdes Cardeal Z. Determination of acrolein in french fries by solid-phase microextraction gas chromatography and mass spectrometry. J Chromatogr A. 2011;1218(21):33326.

23. Zhang J, Sturla S, Lacroix C, Schwab C. Gut Microbial Glycerol Metabolism as an Endogenous Acrolein Source. MBio. 2018;9(1). Epub 2018/01/18.

24. Wang TW, Liu JH, Tsou HH, Liu TY, Wang HT. Identification of acrolein metabolites in human buccal cells, blood, and urine after consumption of commercial fried food. Food Sci Nutr. 2019;7(5):1668-76. Epub 2019/05/30.

25. Abraham K, Andres S, Palavinskas R, Berg K, Appel KE, Lampen A. Toxicology and risk assessment of acrolein in food. Molecular nutrition \& food research. 2011;55(9):1277-90.

26. Stevens JF, Maier CS. Acrolein: sources, metabolism, and biomolecular interactions relevant to human health and disease. Molecular nutrition \& food research. 2008;52(1):7-25.

27. Meyerhardt JA, Niedzwiecki D, Hollis D, Saltz LB, Hu FB, Mayer RJ, et al. Association of dietary patterns with cancer recurrence and survival in patients with stage III colon cancer. JAMA. 
2007;298(7):754-64. Epub 2007/08/21.

28. Faivre J, Bouvier AM, Bonithon-Kopp C. Epidemiology and screening of colorectal cancer. Best Pract Res Clin Gastroenterol. 2002;16(2):187-99. Epub 2002/04/24.

29. Boyle P, Leon ME. Epidemiology of colorectal cancer. Br Med Bull. 2002;64:1-25. Epub 2002/11/08.

30. Wang $\mathrm{HT}$, Chen TY, Weng CW, Yang $\mathrm{CH}$, Tang MS. Acrolein preferentially damages nucleolus eliciting ribosomal stress and apoptosis in human cancer cells. Oncotarget. 2016.

31. Borowicz S, Van Scoyk M, Avasarala S, Karuppusamy Rathinam MK, Tauler J, Bikkavilli RK, et al. The soft agar colony formation assay. Journal of visualized experiments : JoVE. 2014(92):e51998. Epub 2014/11/20.

32. Chung FL, Young R, Hecht SS. Formation of cyclic 1,N2-propanodeoxyguanosine adducts in DNA upon reaction with acrolein or crotonaldehyde. Cancer research. 1984;44(3):990-5.

33. Wang H-T. Effect of Acrolein in DNA Damage, DNA Repair and Lung Carcinogenesis. New York: New York University; 2012.

34. Wang HT, Zhang S, Hu Y, Tang MS. Mutagenicity and sequence specificity of acrolein-DNA adducts. Chem Res Toxicol. 2009;22(3):511-7.

35. Yang IY, Miller H, Wang Z, Frank EG, Ohmori H, Hanaoka F, et al. Mammalian translesion DNA synthesis across an acrolein-derived deoxyguanosine adduct. Participation of DNA polymerase eta in error-prone synthesis in human cells. J Biol Chem. 2003;278(16):13989-94. Epub 2003/02/14.

36. Sanchez AM, Minko IG, Kurtz AJ, Kanuri M, Moriya M, Lloyd RS. Comparative evaluation of the bioreactivity and mutagenic spectra of acrolein-derived alpha-HOPdG and gamma-HOPdG regioisomeric deoxyguanosine adducts. Chem Res Toxicol. 2003;16(8):1019-28.

37. Minko IG, Washington MT, Kanuri M, Prakash L, Prakash S, Lloyd RS. Translesion synthesis past acrolein-derived DNA adduct, gamma -hydroxypropanodeoxyguanosine, by yeast and human DNA polymerase eta. J Biol Chem. 2003;278(2):784-90. Epub 2002/10/29.

38. Yang IY, Chan G, Miller H, Huang Y, Torres MC, Johnson F, et al. Mutagenesis by acrolein-derived propanodeoxyguanosine adducts in human cells. Biochemistry. 2002;41(46):13826-32.

39. Kanuri M, Minko IG, Nechev LV, Harris TM, Harris CM, Lloyd RS. Error prone translesion synthesis past gamma-hydroxypropano deoxyguanosine, the primary acrolein-derived adduct in mammalian cells. $J$ Biol Chem. 2002;277(21):18257-65.

40. Yang IY, Hossain M, Miller H, Khullar S, Johnson F, Grollman A, et al. Responses to the major acroleinderived deoxyguanosine adduct in Escherichia coli. J Biol Chem. 2001;276(12):9071-6.

41. VanderVeen LA, Hashim MF, Nechev LV, Harris TM, Harris CM, Marnett LJ. Evaluation of the mutagenic potential of the principal DNA adduct of acrolein. J Biol Chem. 2001;276(12):9066-70.

42. Kawanishi M, Matsuda T, Nakayama A, Takebe H, Matsui S, Yagi T. Molecular analysis of mutations induced by acrolein in human fibroblast cells using supF shuttle vector plasmids. Mutation research. 1998;417(2-3):65-73.

43. Kehrer JP, Biswal SS. The molecular effects of acrolein. Toxicol Sci. 2000;57(1):6-15. 
44. LoPachin RM, Gavin T, Petersen DR, Barber DS. Molecular mechanisms of 4-hydroxy-2-nonenal and acrolein toxicity: nucleophilic targets and adduct formation. Chem Res Toxicol. 2009;22(9):1499-508.

45. Cai J, Bhatnagar A, Pierce WM, Jr. Protein modification by acrolein: formation and stability of cysteine adducts. Chem Res Toxicol. 2009;22(4):708-16.

46. Kachel DL, Martin WJ, 2nd. Cyclophosphamide-induced lung toxicity: mechanism of endothelial cell injury. J Pharmacol Exp Ther. 1994;268(1):42-6. Epub 1994/01/01.

47. Patel JM, Block ER. Acrolein-induced injury to cultured pulmonary artery endothelial cells. Toxicol Appl Pharmacol. 1993;122(1):46-53. Epub 1993/09/01.

48. Wang $\mathrm{HT}$, Lin JH, Yang $\mathrm{CH}$, Haung $\mathrm{CH}$, Weng $\mathrm{CW}$, Maan-Yuh Lin A, et al. Acrolein induces mtDNA damages, mitochondrial fission and mitophagy in human lung cells. Oncotarget. 2017;8(41):7040621. Epub 2017/10/21.

49. Huang $\mathrm{CH}$, Chen $\mathrm{YT}$, Lin JH, Wang HT. Acrolein induces ribotoxic stress in human cancer cells regardless of p53 status. Toxicol In Vitro. 2018;52:265-71. Epub 2018/07/03.

50. Horton ND, Mamiya BM, Kehrer JP. Relationships between cell density, glutathione and proliferation of A549 human lung adenocarcinoma cells treated with acrolein. Toxicology. 1997;122(1-2):111-22. Epub 1997/09/26.

51. Ramu K, Perry CS, Ahmed T, Pakenham G, Kehrer JP. Studies on the basis for the toxicity of acrolein mercapturates. Toxicol Appl Pharmacol. 1996;140(2):487-98. Epub 1996/10/01.

52. Rudra PK, Krokan HE. Acrolein cytotoxicity and glutathione depletion in n-3 fatty acid sensitive- and resistant human tumor cells. Anticancer Res. 1999;19(1A):461-9. Epub 1999/05/05.

53. Cancer Genome Atlas N. Comprehensive molecular characterization of human colon and rectal cancer. Nature. 2012;487(7407):330-7. Epub 2012/07/20.

54. Strippoli A, Cocomazzi A, Basso M, Cenci T, Ricci R, Pierconti F, et al. c-MYC Expression Is a Possible Keystone in the Colorectal Cancer Resistance to EGFR Inhibitors. Cancers. 2020;12(3). Epub 2020/03/14.

55. Esterbauer H, Schaur RJ, Zollner H. Chemistry and biochemistry of 4-hydroxynonenal, malonaldehyde and related aldehydes. Free radical biology \& medicine. 1991;11(1):81-128. Epub 1991/01/01.

56. Liu JH, Wang TW, Lin YY, Ho WC, Tsai HC, Chen SP, et al. Acrolein is involved in ischemic strokeinduced neurotoxicity through spermidine/spermine-N1-acetyltransferase activation. Experimental neurology. 2020;323:113066. Epub 2019/10/21.

57. Ju WD, Velu TJ, Vass WC, Papageorge AG, Lowy DR. Tumorigenic transformation of NIH 3 T3 cells by the autocrine synthesis of transforming growth factor alpha. The New biologist. 1991;3(4):380-8. Epub 1991/04/01.

58. Xu H, Yu Y, Marciniak D, Rishi AK, Sarkar FH, Kucuk O, et al. Epidermal growth factor receptor (EGFR)related protein inhibits multiple members of the EGFR family in colon and breast cancer cells. Molecular cancer therapeutics. 2005;4(3):435-42. Epub 2005/03/16. 


\section{Figures}

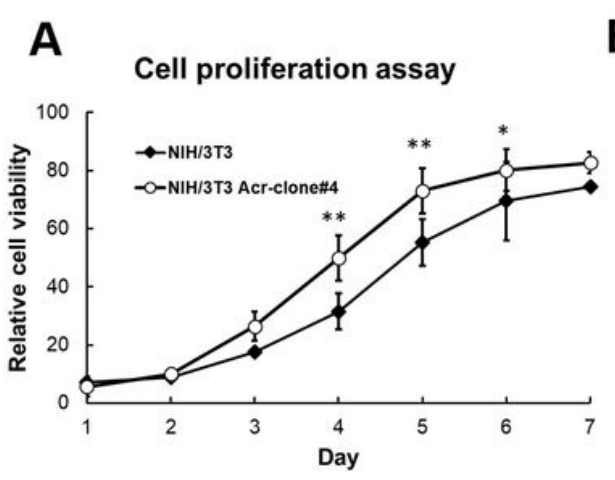

C

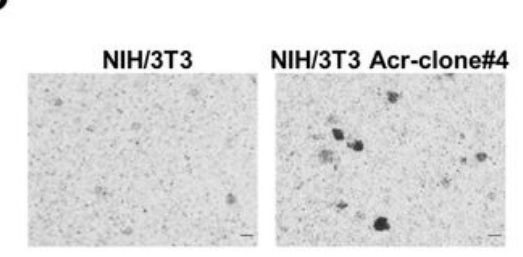

Soft agar colony formation assay

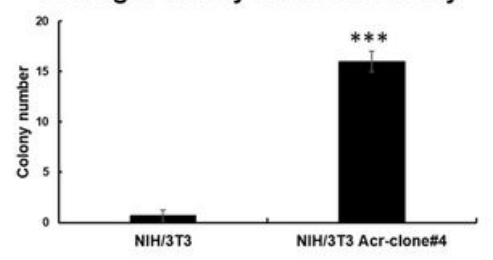

E
B

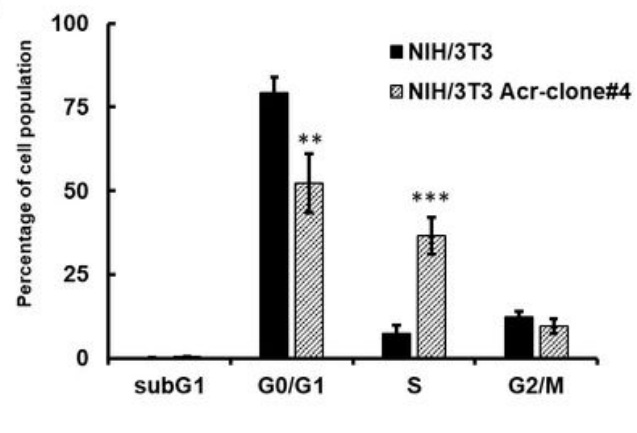

$\mathbf{F}$

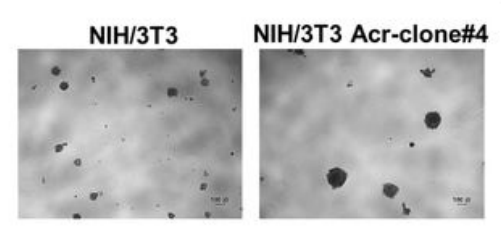

Sphere formation assay

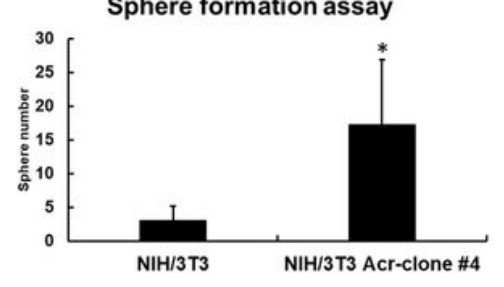

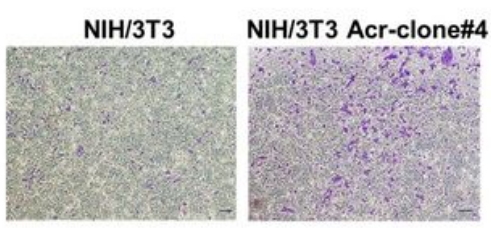

Migration assay

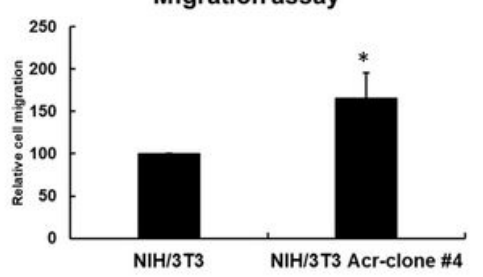

Figure 1

Acrolein induced oncogenic transformation using cellular model, NIH/3T3 cells. NIH/3T3 cells were treated acrolein (Acr, $7.5 \mu \mathrm{M}$ ) for one month and named as NIH/3T3 Acr-clone\#. (A) cell proliferation of $\mathrm{NIH} / 3 \mathrm{~T} 3$ Acr-clone \#4 was analyzed using MTT assays. (B) cell cycle progression of NIH/3T3 Acr-clone \#4 was analyzed using cell cycle analysis with PI staining. (C) Soft agar anchorage dependent cell growth of NIH/3T3 Acr-clone \#4 was analyzed using soft agar assay. (D) Spheroid formation ability of $\mathrm{NIH} / 3 \mathrm{~T} 3$ Acr-clone \#4 was analyzed ultra-low attachment plates (E) Cell migration activity of NIH/3T3 Acr-clone \#4 was analyzed using transwell migration analysis. NIH/3T3 Acr-clone \#4 has the highest cell transformation activity. Student's $t$ tests were used to determine statistical significance, and two-tailed $p$ values are shown. ${ }^{\star} \mathrm{p}<0.05,{ }^{* \star} \mathrm{p}<0.01,{ }^{\star \star *} \mathrm{p}<0.005$ compared with $\mathrm{NIH} / 3 \mathrm{~T} 3$ parental cells. 


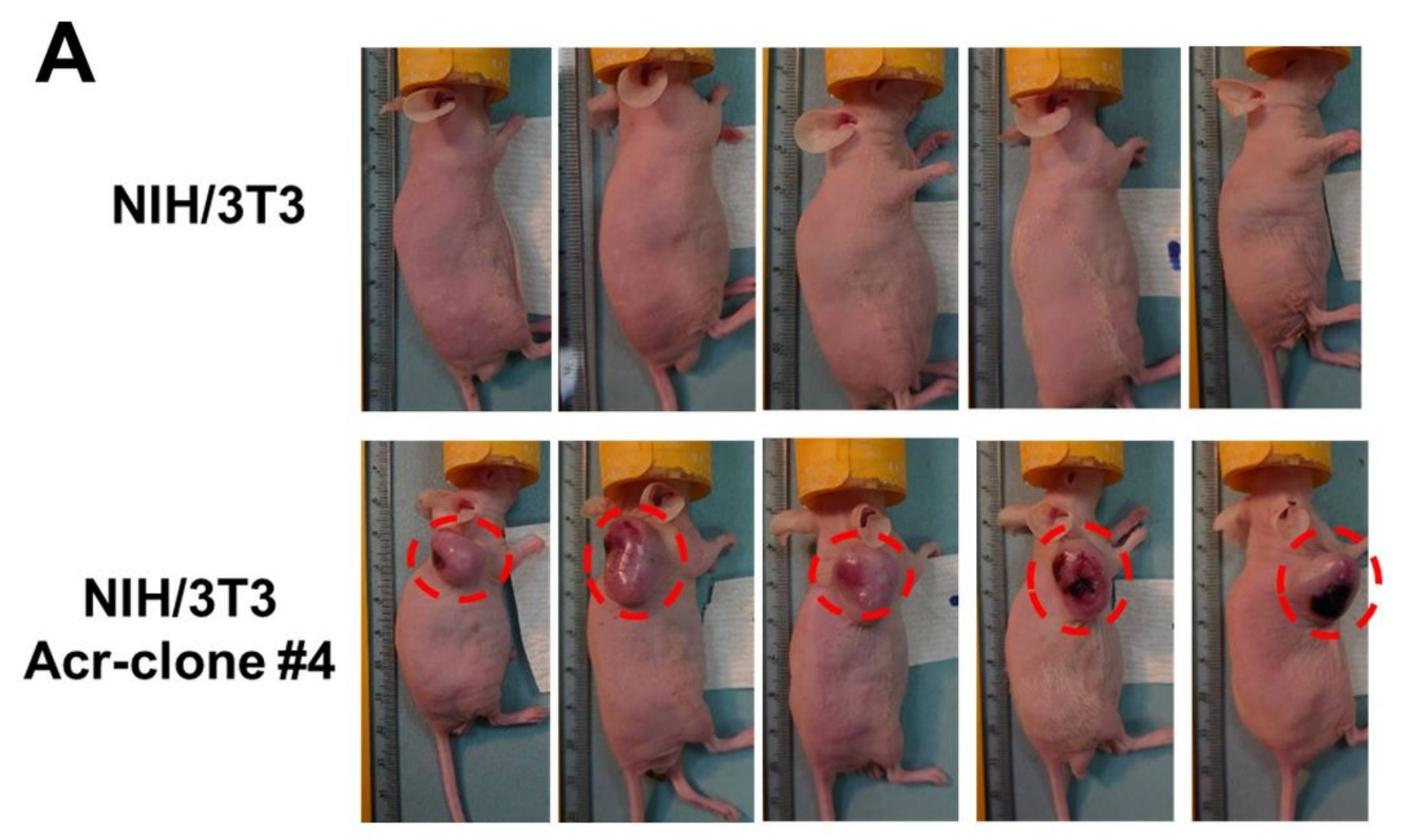

B
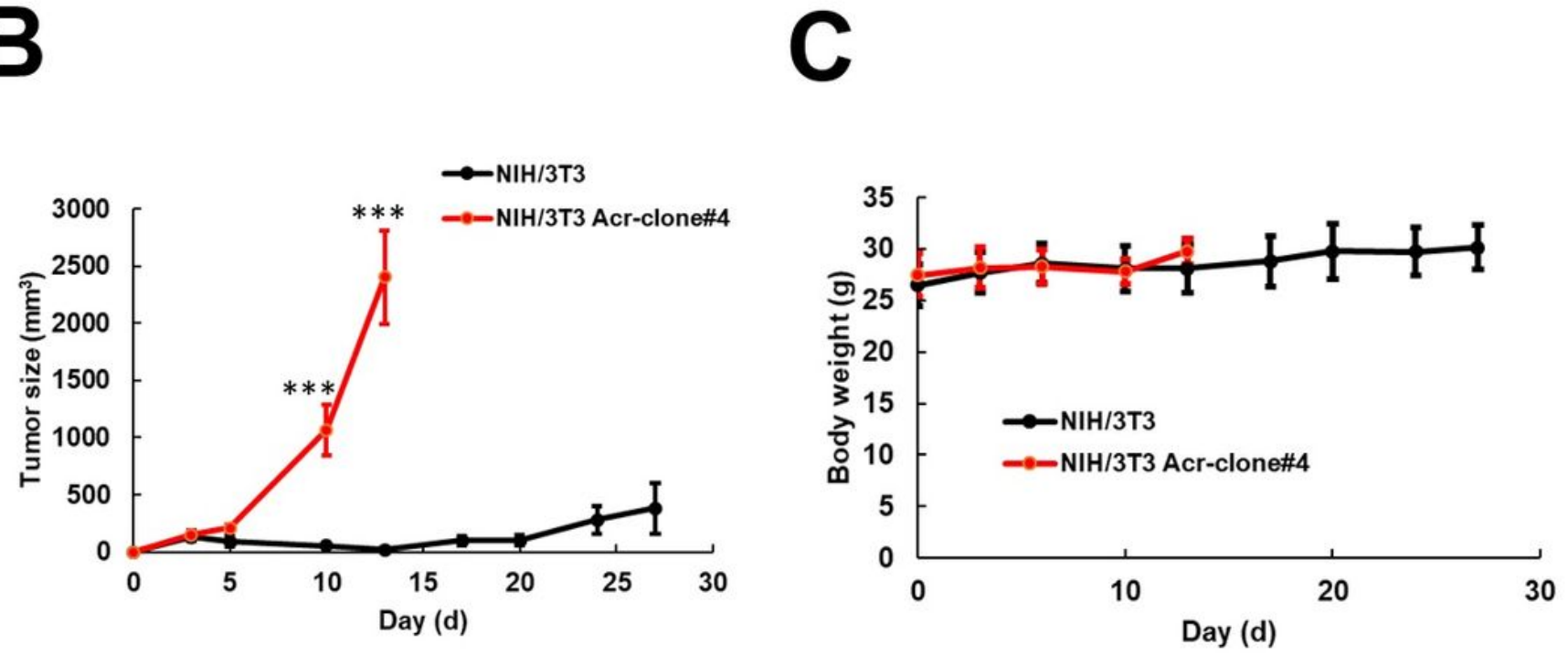

Figure 2

Xenograft mice model of acrolein-transformed clones. (A) Overall view of tumors formed by acroleintransformed NIH3T3 cell clone (Acr-clone\#4). Tumors in nude mice were seen after injection with acrolein-transformed clone\#4, whereas none were seen after injection with mice fibroblast, NIH3T3 cells.

(B) Tumor growth curves and (C) body weight for nude mice of different experimental groups $(n=5)$. Student's $t$ tests were used to determine statistical significance, and two-tailed p-values are shown. *** $\mathrm{p}<0.005$ compared with $\mathrm{NIH} / 3 \mathrm{~T} 3$ parental cells. 

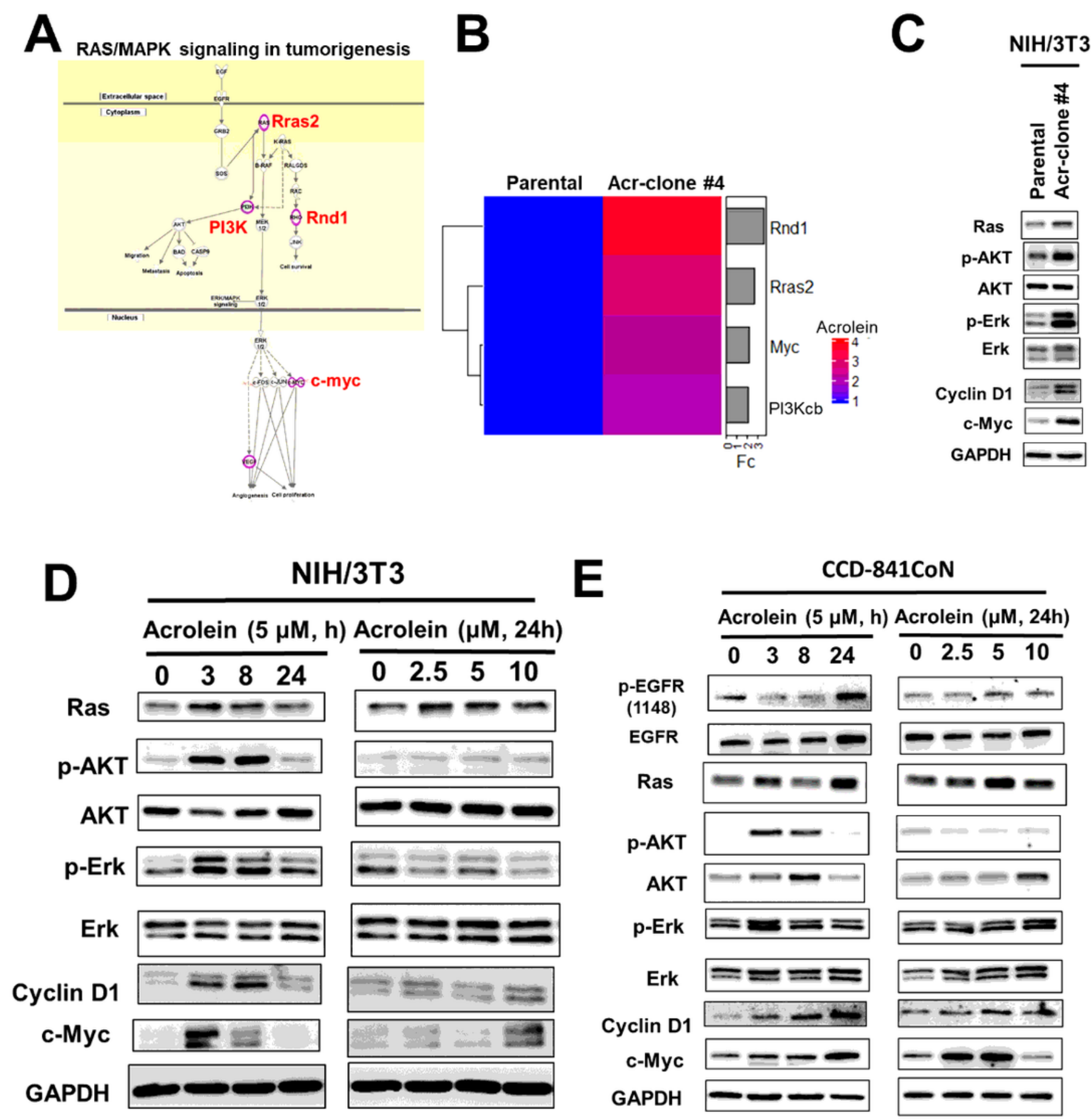

CCD-841CoN
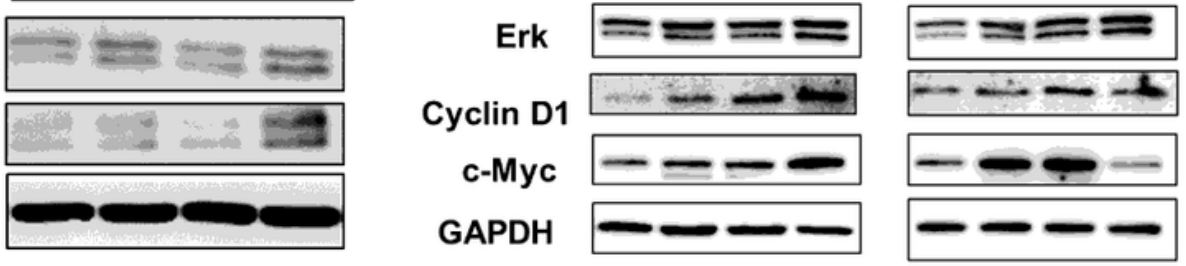

Figure 3

Ingenuity Pathway Analysis (IPA) of gene expression profiles in acrolein-transformed clones. (A) Canonical pathway analysis for gene expression profiles in acrolein-transformed clone\#4 (Acr-clone\#4) using IPA. (B) Heatmap of 4 gene expression (Rnd1, Rras2, myc and PI3Kcb) in acrolein-transformed clone\#4 compared to parental NIH/3T3 cells. (C) Western blot analysis of RAS/ERK and AKT pathway in acrolein-transformed clone\#4 compared to parental NIH/3T3 cells. (D \& E) Dose and time effects of 
acrolein on RAS expression, AKT activation, ERK activation, cyclin D1 and c-myc expression in NIH/3T3 cells (D) and CCD-841CoN cells (E) using western blot analysis. For dose and time effect, cells were treated with different concentrations of acrolein $(0-10 \mu \mathrm{M})$ for $24 \mathrm{~h}$ or acrolein $(5 \mu \mathrm{M})$ for $3-24 \mathrm{~h}$, respectively.

A

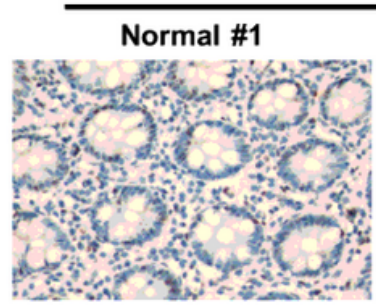

Normal \#2

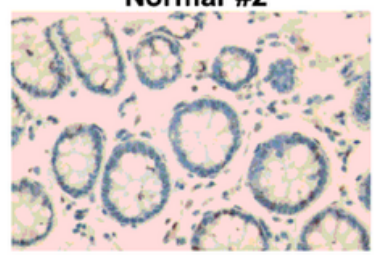

Acrolein-dG

Tumor \#1

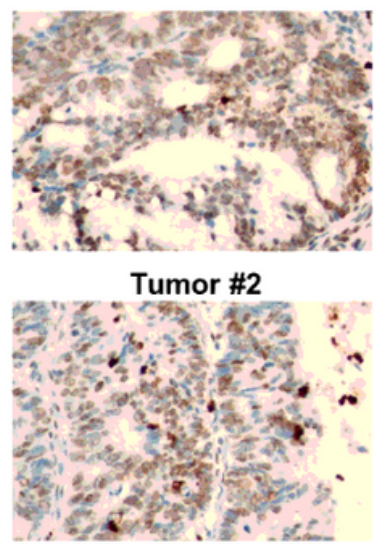

B

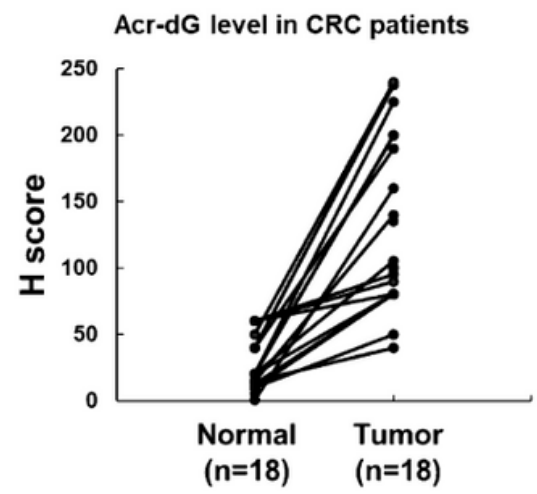

C

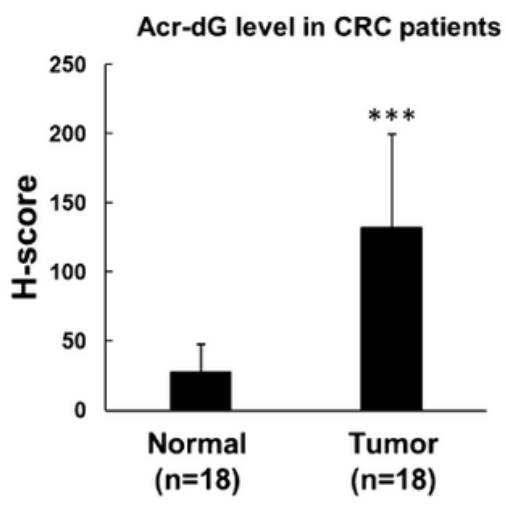

Figure 4

Immunohistochemical staining for Acr-dG adducts in eighteen CRC patients. (A) Representative image of Acr-dG adducts in normal epithelial cells adjacent to the CRC tumor tissues. (B-C) quantification of Acr-dG adducts in normal epithelial cells adjacent to the CRC tumor tissues (magnification, $\times 400$ ). Student's $t$ tests were used to determine statistical significance, and two-tailed $p$-values are shown. ${ }^{\star} p<0.05$, $\star \star p<0.01,{ }^{* \star *} p<0.005$ compared between tumor tissues and normal tissues. 


\section{Overall survival}

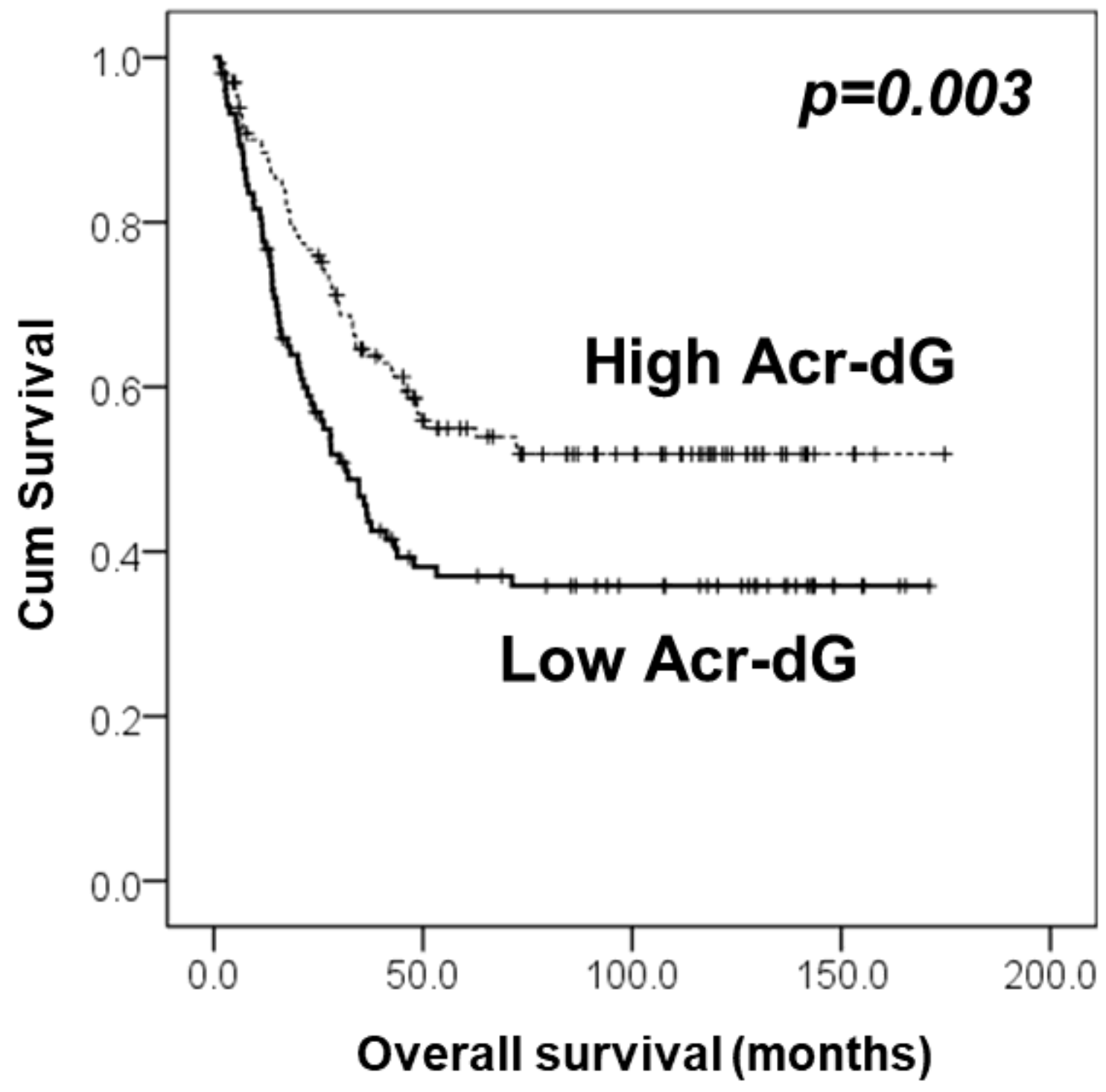

Figure 5

Kaplan-Meier survival analysis of high and low Acr-dG expression group in CRC patients. IHC analysis of Acr-dG levels in CRC tissues ( $n=123)$ were analyzed as described in Materials and Methods. P-value was obtained from the log rank test using SPSS statistical analysis software.

\section{Supplementary Files}

This is a list of supplementary files associated with this preprint. Click to download.

- SupplementaryFigures.docx 